\title{
Heart valve involvement in endomyocardial fibrosis
}

\author{
Geoffr ey Farrer-Brown and M. H. Tarbit \\ From the Bland-Sutton Institute of Pathology, ${ }^{1}$ the Middlesex Hospital Medical School, \\ London; and the Department of Pathology, Makerere University Medical School, \\ Kampala, Uganda
}

The process of involvement of the valves of the heart in endomyocardial fibrosis is described after an injection, microradiographic, and histological study of II hearts with this disease which were part of a larger series of 147 hearts obtained at necropsy from patients dying at the Mulago Hospital, Kampala, Uganda. Using this technique the valves were avascular and not primarily diseased, but were secondarily affected by encroaching endocardial thrombus which pulled the leaflets downwards and bound them down with resulting severe incompetence of the valve in the later stage. The commoner sites of involvement were the posterior leaflet of the mitral valve and the posterior and septal leaflets of the tricuspid valve, while this paper also reports for the first time the uncommon involvement of the pulmonary valve. The manner of involvement of the valves was similar whether this area was affected alone or in combination with ventricular apical lesions.

Involvement of the atrioventricular valves of the heart in endomyocardial fibrosis has been recognized since the first pathological description of this disease by Davies (1948). Diagnosis is relatively simple when the organizing thrombus or fibrous tissue involving the valves is in continuity with lesions in the apices of the ventricles, but confusion with other types of valvular disease may occur when this area alone is involved. In addition, there is often difficulty in diagnosis when there are features suggesting combined rheumatic heart disease. In view of the frequency of both conditions occurring in patients in Uganda (Shaper, Hutt, and Coles, 1968) and the suggestion that endomyocardial fibrosis may be an unusual manifestation of rheumatic heart disease (Shaper, 1966), there is a need for strict criteria to enable pathologists to distinguish the two diseases. In a recent injection, microradiographic, and histological study of 147 'normal' and 'abnormal' hearts obtained at necropsy on patients dying at the Mulago Hospital, Kampala, the appearances of the valves with either or both conditions were studied. The present paper reports the features seen in the endomyocardial fibrosis valves.

Received 17 March 1972.

${ }^{1}$ Present address of both authors.

\section{Materials and method}

A study was made of II hearts which showed the pattern of pathology of endomyocardial fibrosis as described by Shaper et al. (I968). The details of age, sex, tribe, and heart weight of each patient are shown in the Table. The 7 male and 4 female patients had an age range of 5 to 60 years, with a median of 20 years. The weights of the hearts varied from 220 to $520 \mathrm{~g}$. When related to the body weight of the patient (Coles and Davies, 1959) 7 of these hearts were considered overweight while 4 were within the normal range, though 3 of them were at the upper limit. The tribal origin of the patients varied and con-

TABLE Details of patients

\begin{tabular}{lllll}
\hline $\begin{array}{l}\text { Case } \\
\text { No. }\end{array}$ & Age & Sex & Tribe & $\begin{array}{l}\text { Heart } \\
\text { weight } \\
(g)\end{array}$ \\
\hline K6 & I8 & M & Rwanda & 360 \\
K27 & I6 & M & Rwanda & 520 \\
K31 & 60 & M & Luhya & 360 \\
K48 & 50 & M & Rwanda & 300 \\
K73 & I8 & F & Ganda & 520 \\
KI02 & 22 & F & Ganda & 320 \\
KI04 & 20 & M & Hangaza & 450 \\
KI40 & 8 & M & Ha & 390 \\
KI55 & 5 & M & Rwanda & 220 \\
KI66 & 38 & F & Ganda & 310 \\
KI7I & 30 & F & Rwanda & 360 \\
\hline
\end{tabular}


sisted of 3 Ganda and 8 'immigrants' who came from Rwanda or its vicinity.

The basic technique used for this investigation has been described previously (Farrer-Brown, 1968) but a few minor modifications were used in this study, and briefly it is as follows. Radiopaque medium (Colorpaque, Pilot Chemical Ltd), with 4 per cent added gelatin, was injected simultaneously into both coronary arteries at a pressure equivalent to the patient's systolic blood pressure or, if this was unknown, at $120 \mathrm{mmHg}$. After fixation the hearts were sectioned on a bacon slicer from apex to base into uniform $5 \mathrm{~mm}$ thick slices up to just below the mitral valve. The - transventricular slices were $x$-rayed and examined as previously described while the atrial portions of the hearts, submerged in water, were $x$-rayed on to Crystallex film (Kodak) using a watercooled Machlett $x$-ray tube with exposures of $25 \mathrm{kV}$ and $25 \mathrm{~mA}$ for 20 minutes with a tube distance of $64 \mathrm{~cm}$. Each valve was then examined, cut across, and opened out, after which both

t diseased and normal valves were excised and $x$-rayed onto Kodak Maximum Resolution plates using the Machlett $x$-ray tube and exposures of $25 \mathrm{kV}$ and 10 to $20 \mathrm{~mA}$ for 15 to 30 minutes according to the thickness of the slice. Sections across all valves were then processed by conventional histological techniques and stained by haematoxylin and eosin and elastic van Gieson.

,

\section{Results}

All I I hearts in this study showed valve disease. In 7, endomyocardial fibrosis affected the mitral valve, in 7 the tricuspid, and in 2 the pulmonary valve. A range of patho- logical appearances from the earliest to late stages was seen and the disease process was the same whether the valve region was involved by endomyocardial fibrosis in isolation or in continuity with disease of the apices of the heart.

Early valve involvement is shown in Fig. I where thrombus is present on the thickened and fibrotic upper end of the posterior papillary muscle of a mitral valve with the chordae tendineae beginning to be involved and pulled downwards. Organizing thrombus may then extend up along the whole length of the chordae tendineae to the valve leaflet before the adjacent myocardial endocardium is involved (Fig. 2). The papillary muscle becomes tethered to the myocardial wall with consequent restriction of valve mobility (Fig. 3). The organizing thrombus on the myocardial endocardium then extends upwards to involve the undersurface of the valve. Fig. 4 is a microradiograph of a cross-section of an uninvolved, avascular, mitral valve to show that the encroaching thrombus is partly

- vascularized with many small vessels extending out in an irregular manner from the sub-

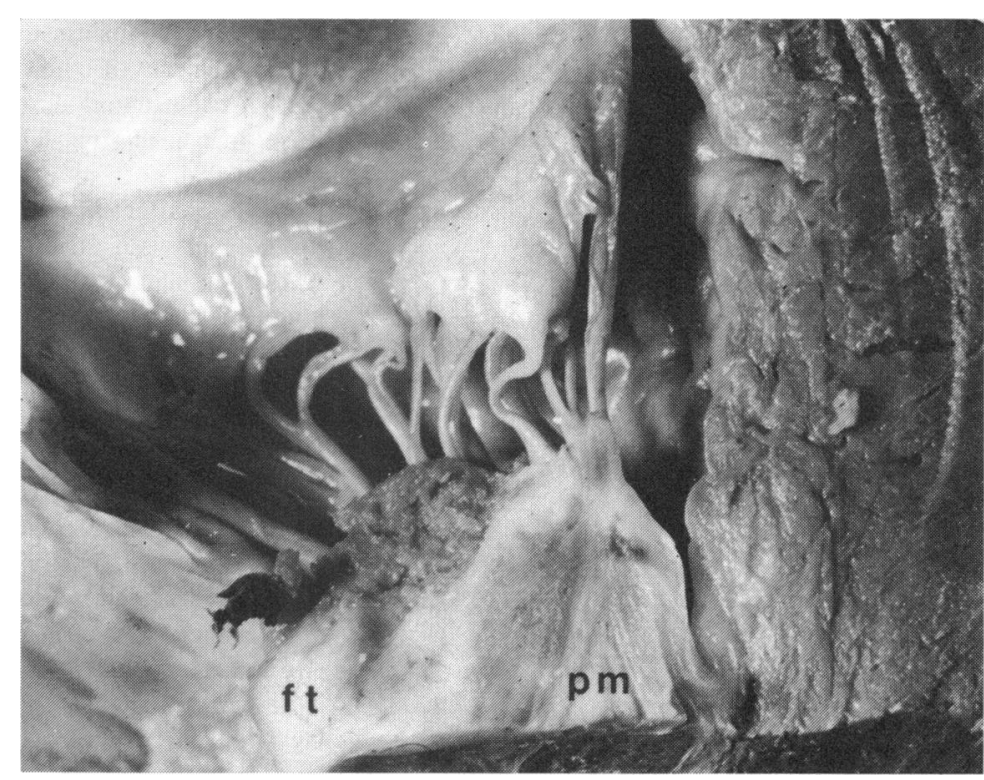

FIG. I Thrombus present on the thickened and fibrotic upper end of a posterior papillary muscle (pm) with the chordae tendineae beginning to be pulled down. ( $f t=$ fibrous tissue.) $(\times 6$.

endocardial area into the thrombus. This abnormal vascular area outlines the zone of organization of the thrombus with the remainder still being cellular and avascular. The undersurface of the atrioventricular valves then becomes involved initially at the valve ring (Fig. 5) but later along the whole or majority of their length. Histology shows that at this stage radiopaque filled vessels are present on the upper surface of the thrombus (Fig. 6) but the valve itself contains no vessels or evidence of inflammation. The shape of this valve is comparatively normal but subsequent shrinkage of the attached thrombus after organization would later pull the valve down towards the myocardial endocardium.

Two hearts with extensive organizing thrombus filling the majority of the right ventricle showed this thrombus involving the underside of the pulmonary valve. Fig. 7, a haematoxylin and eosin section, shows the vascularity of the organizing thrombus on the myocardial endocardium and the undersurface of the valve, but the normality of the valve leaflet itself is more easily visualized with an elastic van Gieson stain and the radiopaque medium dissolved out (Fig. 8). Fig. 9 shows an area of the second involved 


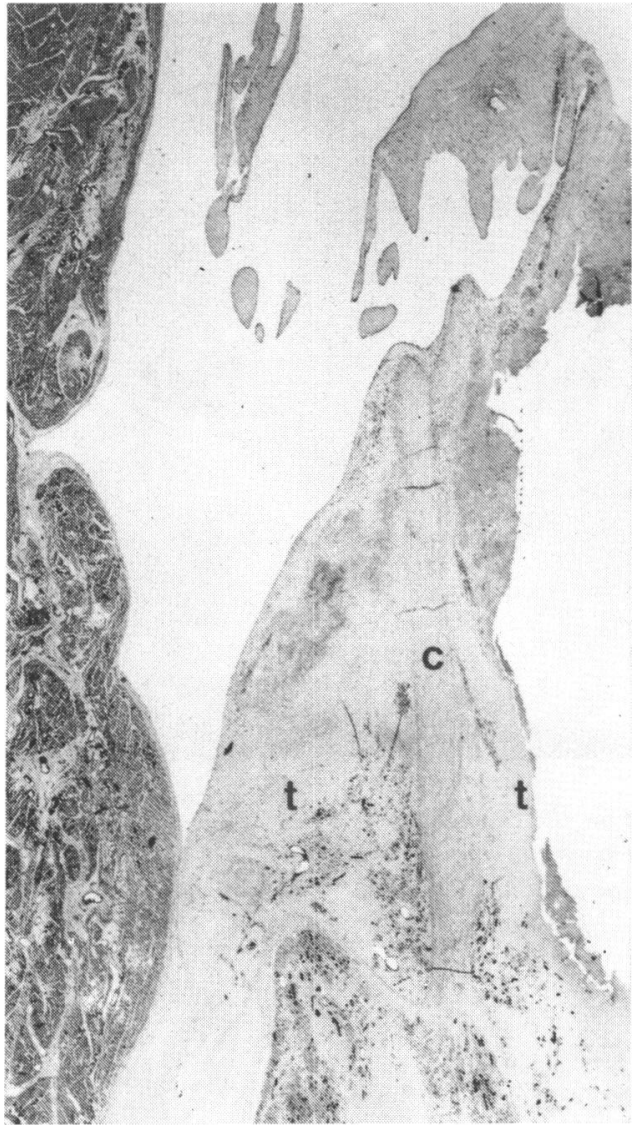

FIG. 2 Organizing thrombus $(t)$ extending up along the whole length of the chordae tendineae $(c)$, before the adjacent myocardial endocardium is involved. ( $\times 6$.

pulmonary valve, where the thrombus just encroached onto the valve, but in addition below this it illustrates the rounded surface and layering of organizing thrombus associated with blood flowing through the pulmonary valve.

The late stage of involvement of the mitral valve by endomyocardial fibrosis was seen in two hearts from children aged 5 and 8 years. Complete organization of the thrombus with replacement by fibrous tissue had resulted in shrinkage and distortion of the posterior cusp of the valve (Fig. IO). In each case this leaflet had been pulled downward and towards the myocardial endocardium so that only a 2-3 $\mathrm{mm}$ ridge remained (Fig. I I). The inferior view of the second valve (Fig. 12) illustrates the valve incompetence and the characteristic binding down of the chordae tendineae onto the myocardial endocardium with the fibrous tissue encroaching onto the undersurface of the valve with consequent loss of the normal sharp line of junction of the base of the valve with the wall. Histology (Fig. 13) of this severely diseased valve shows fairly vascular fibroelastic tissue filling the angle between the myocardium and the undersurface of the valve. Even in this late stage of the disease the injection studies have not shown any small arteries within the valve itself (Fig 14).

Three hearts in this study showed combined endomyocardial fibrosis and rheumatic heart disease. Features of the latter disease were seen in the mitral and aortic valves of all 3 hearts and in the tricuspid valve of one. A single mitral valve showed the posterior cusp affected by endomyocardial fibrosis and the anterior part by rheumatic heart disease. The vascularity of the valve areas affected by the two diseases differed. In contrast to the absence of vessels in valves in endomyocardial fibrosis, those in rheumatic heart disease showed conspicuous vascularity, as shown in Fig. 15, the excised anterior portion of a mildly affected rheumatic valve, and in Fig. 16 the microradiograph of the same valve.

\section{Discussion}

The process of involvement of the valves in hearts affected by endomyocardial fibrosis is basically similar whether the valve area is affected alone or in combination with apical lesions. This disease which mainly results in endocardial fibrosis, but also causes myocardial scarring, does not primarily affect heart valves. The initial process whereby the endocardium of the ventricles is damaged is unknown, but the finding that the thrombus which is laid down may encroach on the undersurface of the valves, with resulting distortion, is in agreement with the descriptions of Davies (1948) and Connor et al. (1967, 1968). The valves become bound down by fibrous tissue so that finally only a rim of the valve appears to remain. In fact the structure of the valve leaflet can still be discerned microscopically at the top edge of the organized thrombus or fibrous tissue. The present injection study has shown that the valve is avascular and in itself is not diseased nor does it contain an inflammatory cell infiltrate.

The part of the valve affected is determined by the extent of underlying endocardial thrombus which must be thick enough to encroach onto the valve. Consequently, the first site of mitral valve involvement is likely to be superior to the posterior papillary muscle where the chordae tendineae become 

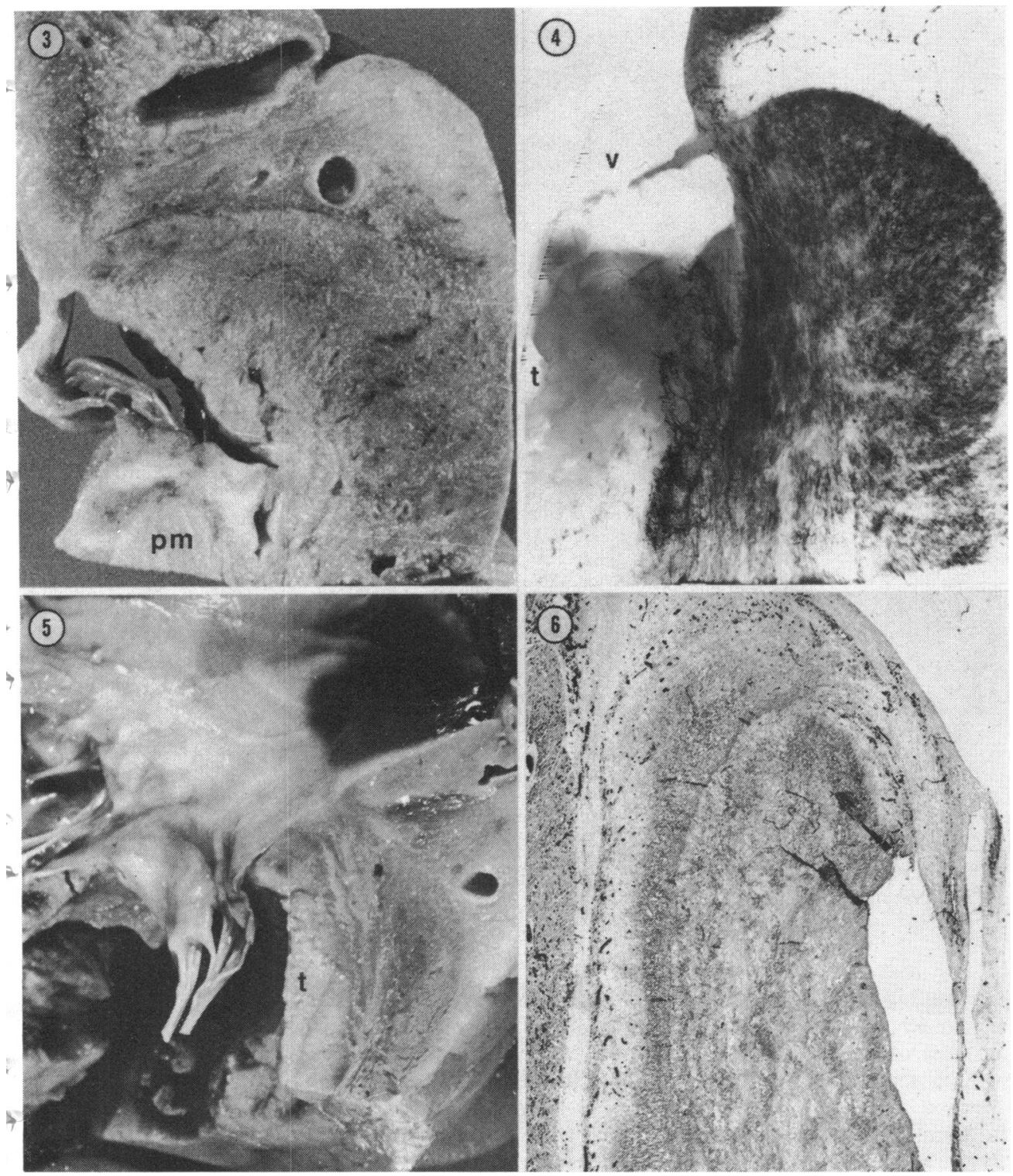

FIG. 3 Section across a posterior papillary muscle ( $p m$ ) tethered to the myocardial wall shows how valve mobility may be restricted. $(\times 4$.

- FIG. 4 Microradiograph of a section across a normal avascular mitral valve (v) with partially - vascularized endocardial thrombus $(t)$ extending up close to the cusp. $(\times 3$.

FIG. $5 A$ cross-section of the posterior cusp of a tricuspid valve with endocardial thrombus $(t)$ extending onto the undersurface of the valve. $(\times 4$.

FIG. 6 Histological section of the specimen shown in Fig. 5 illustrating small vessels filled with radiopaque medium on the upper surface of the thrombus on the undersurface of the valve cusp. (H. and $E . \times 9$. 


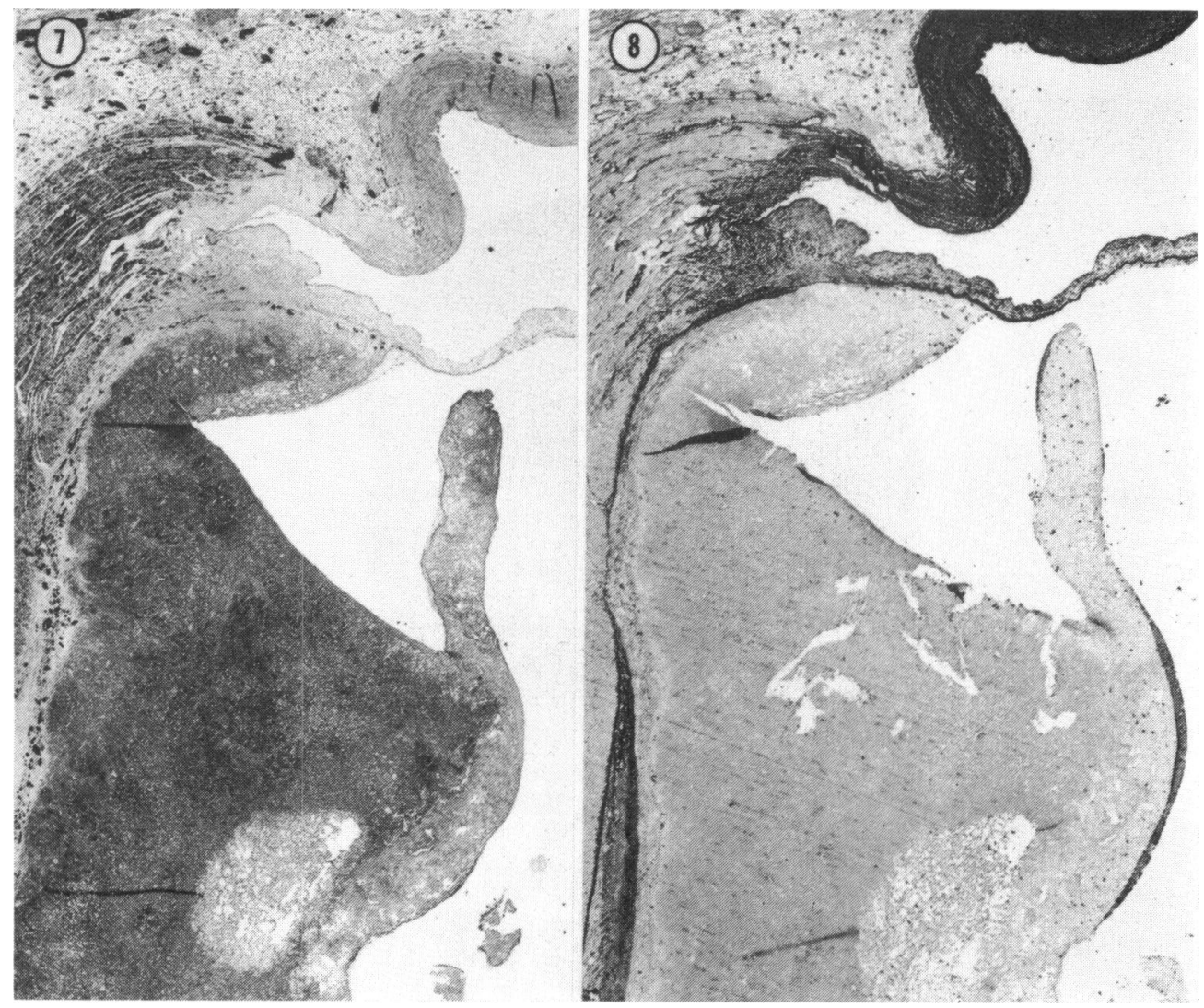

FIG. 7 A cross-section of a pulmonary valve showing thrombus extending onto the undersurface. ( $H$. and $E . \times I I$.)

FIG. 8 An elastic Van Gieson stain of the same section as Fig. 7 to show the outline of the normal valve cusp. Radiopaque medium has been dissolved out. ( $\times$ I I.)

bound down which results in pulling down of the valve itself towards the upper surface of the thrombus. Usually the area of mitral valve involved, even in the late stages, is confined to the posterior portion with its close proximity to the endocardium while the anterior part of the mitral valve situated some distance from the endocardium is unlikely to be reached by the thrombus. Similarly, the region affected in the tricuspid valve is that part close to the endocardial surface, and usually the posterior and septal leaflets. The subsequent distortion of this valve is not usually as gross as the mitral valve probably because the leaflets are longer and more mobile, but the resulting incompetence may be just as severe clinically as when the mitral valve is involved. Because of the above disease process stenosis of these valves does not occur in endomyocardial fibrosis.

The involvement of the pulmonary valve in endomyocardial fibrosis has not been described previously but it was present in two hearts in this study with extensive thrombus in the right side of the heart. It is presumably unusual for this to happen as flow of blood past the pulmonary valve is sufficiently fast to prevent significant thrombus formation. The curved upper surface and layering of the thrombus just below this valve in one of the hearts is indicative of the flow of blood affecting the formation of the th:ombus just as in any thrombosing artery. No convincing report of the aortic valve being involved in endomyocardial fibrosis has been traced. As the heart valves are not primarily diseased in endomyocardial fibrosis valvular bacterial endocarditis is unlikely to occur. However, emboli causing systemic disturbances may still result from mural thrombi, as seen in one patient in this series.

Shaper et al. (1968) have referred to the 
FIG. 9 A section of an involved pulmonary valve with the thrombus below showing a rounded surface and layering associated with blood flow through the valve. $(H$. and $E$. $\times$ Io.)

FIG. Io The shrunken distorted posterior cusp of a mitral valve seen in the late stage of the disease. Part of the anterior cusp is seen on the right of the Fig. $(\times 2$.

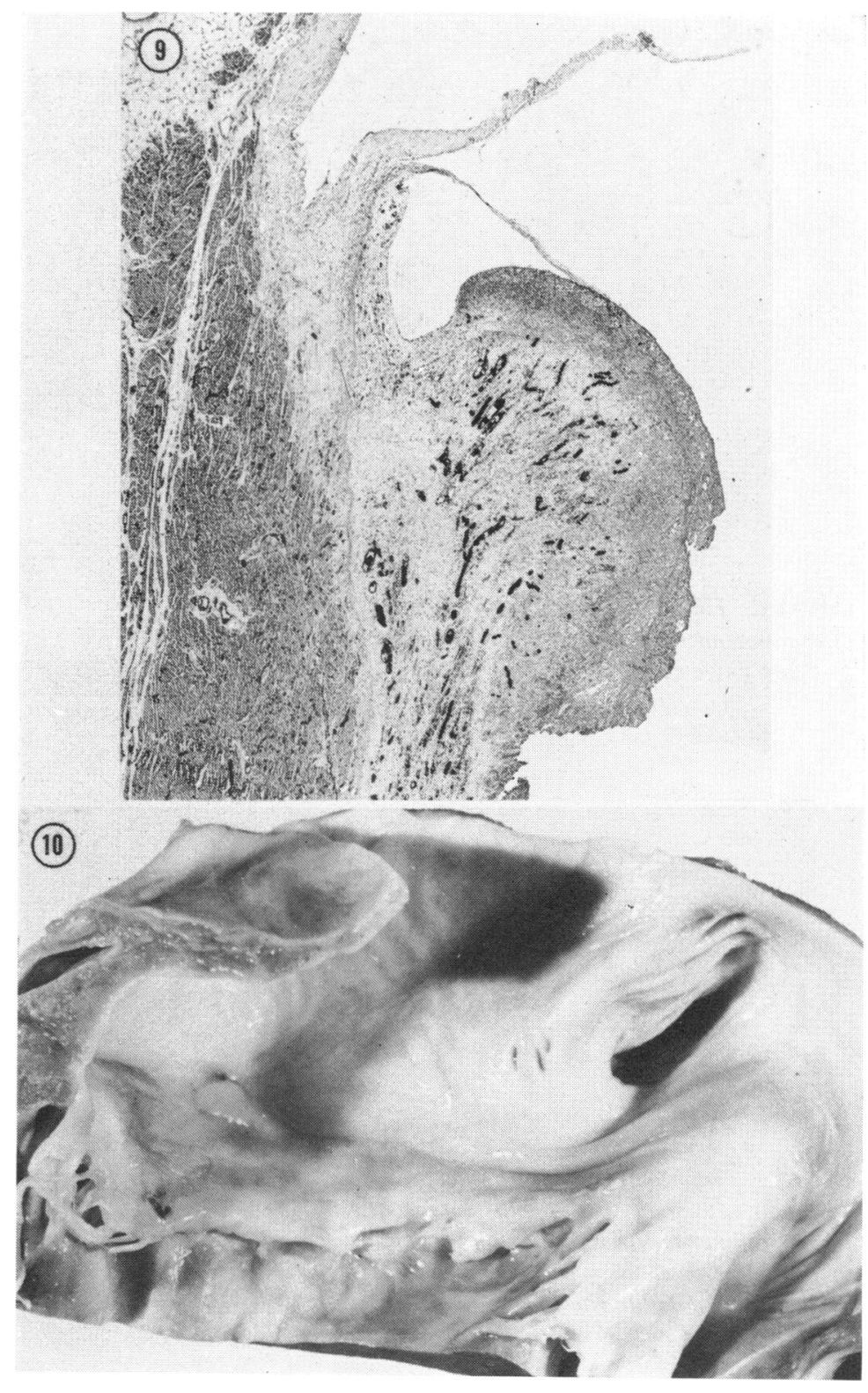


FIG. I I High power of the posterior cusp of another mitral valve to show that only a 2-3 $\mathrm{mm}$ ridge remains. $(\times 5$.)

FIG. I 2 Inferior view of the same valve as in Fig. II, to show the incompetence, with loss of the normal sharp junction of the base of the valve with the wall, plus binding down of the chordae tendineae by endocardial fibrous tissue. $(\times 3.5$.
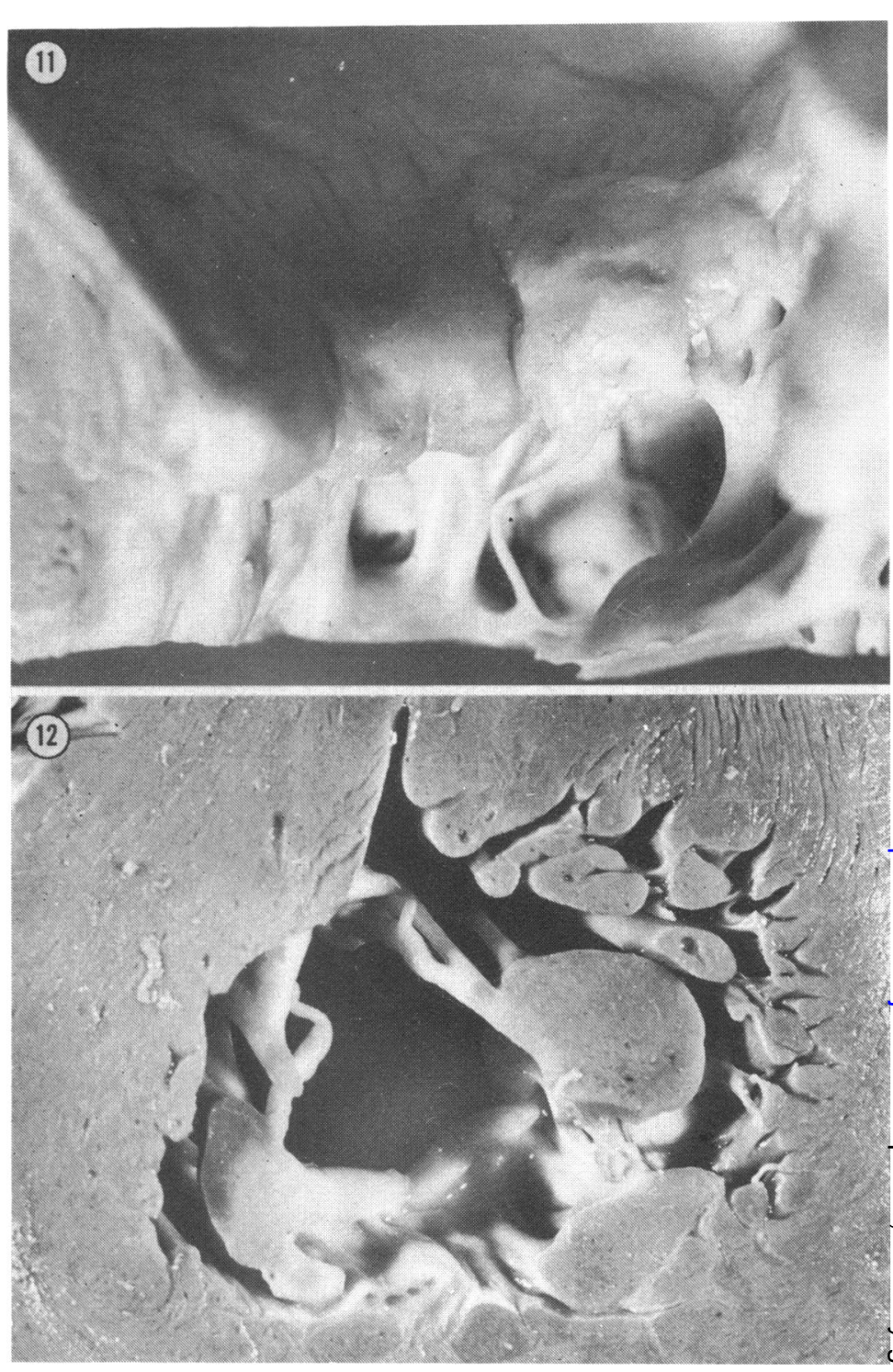
FIG. I 3 Histology of the same valve as in Fig. II and 12 with fairly vascular fibroelastic tissue filling the angle between the ventricle wall and the valve cusp (v). (Elastic 'van Gieson. × 9.)
(13)
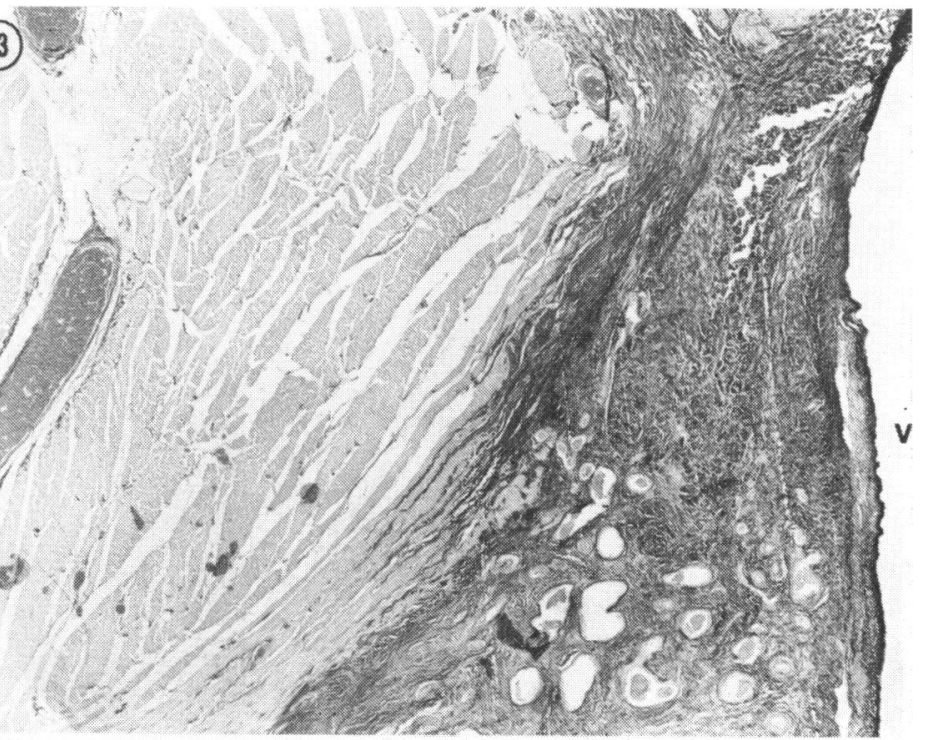

(14)

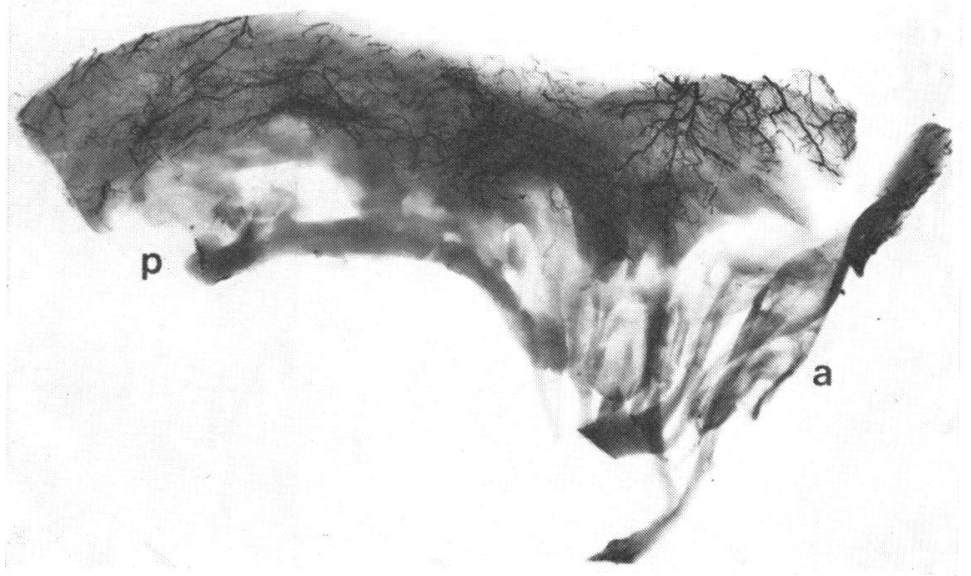

- FIG. I 4 Microradiograph of the same valve as in Fig. Io to show that the distorted and fibrotic posterior leaflet $(p)$ is avascular as well as the normal anterior leaflet $(a) .(\times 4$. 
FIG. I 5 The excised anterior leaflet of a mitral valve mildly affected by rheumatic heart disease. Small vessels can be seen within the valve, with the most dense vascularity being between the two arrows. $(\times 2$.

FIG. I 6 Microradiograph of the arrowed area of the valve shown in Fig. I 5 illustrating the obvious vascularity of this valve. $(\times 4.5$.)
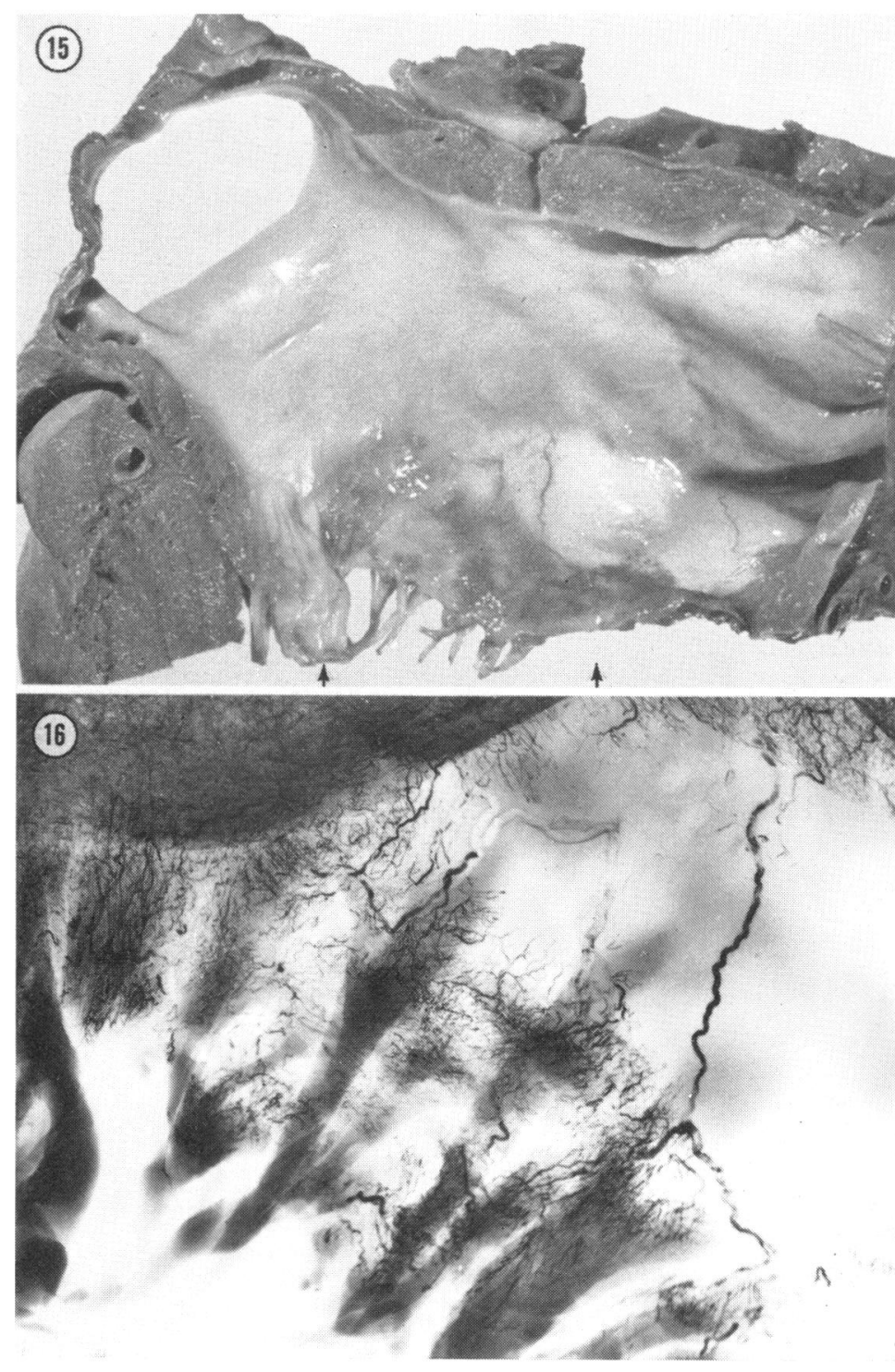
unusual coincidence of rheumatic heart disease and endomyocardial fibrosis in the same heart. However, it is often difficult to be certain whether the valves are affected by endo- myocardial fibrosis or rheumatic heart disease or by both conditions. The distribution of the

- disease and the part and manner in which the valve is affected may be sufficient to differentiate, but viewing the valve inferiorly has proved an added advantage. The binding down of the chordae tendineae, always present - in endomyocardial fibrosis even in mildly affected valves, is best viewed in this position $\exists$ and is not present in rheumatic heart disease, though the chordae may be thickened and vascularized. If there is still uncertainty after gross examination, the microscopy will reveal a normal valve in endomyocardial fibrosis $x$ though this may be indistinct on haematoxylin and eosin sections and may require staining 7 with an elastic van Gieson stain to obtain good demarcation from the thrombus. The - vessels in the valve will be present only in the thrombus in endomyocardial fibrosis whereas the vessels in rheumatic heart disease will be within the valve itself (FarrerBrown and Tarbit, 1972) which in addition will show other histological features of primary valve disease.

This study was carried out with the close cooperation of Professors M. S. R. Hutt, R. A. B. Drury, and $\mathrm{K}$. Somers. We are extremely grateful to them and to Professor G. Dick, Dr. A. G. Shaper, and Dr. A. Pomerance for their constant zadvice and helpful criticism. We thank the many physicians and pathologists who provided material and Mrs. P. D. James, Mrs. I. Steiner, Mrs. R. Coles, Miss E. Moss, and Miss J. Whittingham for their technical help, and Professor A. C. Thackray for assistance with the histological photographs. This work was supported by a grant from the British Heart Foundation.

\section{References}

Coles, R. M., and Davies, J. N. P. (1959). The normal heart weight of Uganda Africans. East African Medical fournal, 36, 76.

Connor, D. H., Somers, K., Hutt, M. S. R., Manion, W. C., and D'Arbela, P. G. (1967). Endomyocardial fibrosis in Uganda (Davies' disease) Part I. American Heart fournal, 74, 687.

Connor, D. H., Somers, K., Hutt, M. S. R., Manion, W. C., and D'Arbela, P. G. (1968). Endomyocardial fibrosis in Uganda (Davies' disease) Part II. American Heart fournal, 75, 107.

Davies, J. N. P. (1948). Endocardial fibrosis in Africans. East African Medical Fournal, 25, 10.

Farrer-Brown, G. (1968). The injection of capillaries, arterioles, and arteries in the ventricles of the human heart by a radio-opaque medium. Cardiovascular Research, 2, 179.

Farrer-Brown, G., and Tarbit, M. H. (1972). The microvasculature of heart valves in rheumatic heart disease. American Heart fournal. In the press.

Shaper, A. G. (1966). Endomyocardial fibrosis and rheumatic heart disease. Lancet, $1,639$.

Shaper, A. G., Hutt, M. S. R., and Coles, R. M. (1968). Necropsy study of endomyocardial fibrosis and rheumatic heart disease in Uganda 1950-1965. British Heart fournal, 30, 391.

Requests for reprints to Dr. Geoffrey FarrerBrown, Bland Sutton Institute of Pathology, The Middlesex Hospital Medical School, London W.I. 